\title{
Stability of electronic states of the vacancy in diamond
}

\author{
Alison Mainwood $\dagger$ and A M Stoneham $\ddagger$ \\ $\dagger$ Physics Department, King's College London, Strand, London WC2R 2LS, UK \\ $\ddagger$ Centre for Materials Research and Department of Physics and Astronomy, University College \\ London, Gower Street, London WC1E 6BT, UK \\ Received 10 September 1996
}

\begin{abstract}
The vacancy in diamond is a fundamental defect which has been studied theoretically and experimentally for forty years. However, although early theories (Coulson C A and Kearsley M J 1957 Proc. R. Soc. A 241 433) were extremely successful in explaining the nature of the ground state of the neutral defect and the Jahn-Teller distortion expected (Lannoo M and Stoneham A M 1968 J. Phys. Chem. Solids 29 1987), there are still several questions which have not been answered satisfactorily. In particular, the many-electron effects and configuration interaction are vital. They determine not only the order of electronic levels in the vacancy, but also the best-known optical transition, GR1, which cannot be expressed in terms of one-electron levels alone.

We bring together much of the detailed recent experimental data on the different charge states and excited states of the vacancy to build up a simple empirical model of the defect. We show that the stability of the states and their photoconductivity, or lack of it, can be reproduced. We can predict that other states of the neutral vacancy, observable by EPR, lie very close above the ground state, and another high-energy optical transition might be detectable.
\end{abstract}

\section{Introduction}

Optical spectroscopy has been the major experimental tool for studies of diamond [1]. The wide bandgap and the wealth of sharp spectral features from intrinsic defects have encouraged studies of the effects of applied fields [2], and hence of the Jahn-Teller effect [3]. Spin resonance has begun to come into its own as a major tool with the synthesis of diamonds with suitable isotopic compositions [4]. The emphasis on optical spectra has led theorists to stress optical properties of simple defects, so excited-state energies are important as well as ground states. This leads to a different perspective from that of most local density functional work [5], which has concentrated much more on the ground-state energies.

The pioneering studies of the vacancy [6] remain remarkably successful, even after nearly 40 years. Likewise, the current views of the Jahn-Teller effect are very close to those of Lannoo and Stoneham [7] expressed in 1968. However, there has been very little theory of the relative energies involved in changing the charge state, so there is significant confusion about photoionization energies, though some guidance can be had from the work of Bernholc et al [8]. Questions like 'where is the vacancy ground state in the gap?' can also confuse, since the one-electron description which this question sometimes implies is not appropriate. Indeed, the best-known transition of the vacancy (the GR1 line) cannot be described as a one-electron transition [6]. This transition energy (1.673 eV for the zerophonon line) is independent of the splitting of one-electron states, to first order, and is actually determined by the strong electron-electron interactions. 
In recent years, a body of evidence has grown up which does allow one to put reasonably tight bounds on key energies, both the ground- and excited-state energies, including a comparison between all charge states of the defect. This we shall demonstrate, using as a framework the analysis of Lannoo [9]. This approach is a simple one which is intended to allow to emerge the intuitive understanding of a complex many-electron system.

Table 1. The energies of the observed electronic states of the vacancy. The CI values are calculated from the parameters derived in section 4, and higher-energy states not listed here.

\begin{tabular}{lllllc}
\hline Charge & State & Configuration & $n$-electron shift & Energy & CI (eV) \\
\hline $\mathrm{V}^{+}$ & ${ }^{2} \mathrm{~T}_{2}$ & $\mathrm{a}^{2} \mathrm{t}$ & $3 \bar{E}+3 \bar{J}$ & $-5 \Delta / 4-U / 4$ & -0.36 \\
$\mathrm{~V}^{0}$ & ${ }^{1} \mathrm{E}$ & $\mathrm{a}^{2} \mathrm{t}^{2}$ & $4 \bar{E}+6 \bar{J}$ & $\Delta-3 U / 4$ & -0.63 \\
$\mathrm{~V}^{0}$ & ${ }^{1} \mathrm{~T}_{2}$ & $\mathrm{a}^{2} \mathrm{t}^{2}$ & $4 \bar{E}+6 \bar{J}$ & $\Delta-U / 4$ & -0.01 \\
$\mathrm{~V}^{0}$ & ${ }^{5} \mathrm{~A}_{2}$ & $\mathrm{at}^{3}$ & $4 \bar{E}+6 \bar{J}$ & $-3 U / 2$ & 0 \\
$\mathrm{~V}^{0}$ & ${ }^{3} \mathrm{~T}_{1}$ & $\mathrm{a}^{2} \mathrm{t}^{2}$ & $4 \bar{E}+6 \bar{J}$ & $\Delta-3 U / 4$ & -0.44 \\
$\mathrm{~V}^{-}$ & ${ }^{4} \mathrm{~A}_{2}$ & $\mathrm{a}^{2} \mathrm{t}^{3}$ & $5 \bar{E}+10 \bar{J}$ & $-3 \Delta / 4-3 U / 2$ & 0 \\
$\mathrm{~V}^{-}$ & ${ }^{4} \mathrm{~T}_{1}$ & $\mathrm{at}^{4}$ & $5 \bar{E}+10 \bar{J}$ & $+\Delta / 4-3 U / 2$ & 0 \\
\hline
\end{tabular}

\section{Framework}

The Lannoo theory [9] follows Coulson and Kearsley [6] in considering the electronic structure of the vacancy in diamond as being simple combinations of the four danglingbond $\mathrm{sp}^{3}$ orbitals. From symmetry arguments they form one-electron $\mathrm{a}_{1}$ and $\mathrm{t}_{2}$ states split by an energy $\Delta$ about a mean energy of $\bar{E}$ measured relative to the valence band edge. Lannoo then calculates the energies of the many-electron states in terms of the dangling-bond electron-electron interaction parameter, $U$ and an exchange parameter $\bar{J}$. In each charge state, there may be several many-electron states of the same symmetry and spin, so a further calculation is necessary to include configuration interaction (CI) within this simple model. Lannoo further discusses the estimates of these parameters derived analytically from Slater orbital integrals, or scaled systematically from them. To illustrate the relative energies of many-electron states within this model, table 1 lists the lowest few states in positive $\left(\mathrm{V}^{+}\right)$, neutral $\left(\mathrm{V}^{0}\right)$, and negative $\left(\mathrm{V}^{-}\right)$charge states in terms of the four parameters and, in the last column, the CI calculated from the values of $\Delta$ and $U$ derived in section 4 (using higher-energy states not included in this table). Lannoo tabulates all of the energies very clearly, and includes the CI in graphs which show the lowest-energy states of the negative, neutral and positive vacancy relative to their energies in the limit of zero electron-electron interaction. Note that one must include shifts of $-3 U / 4,-3 U / 2$, and $-3 U / 2$ in his graphical data, before they can be used to compare the energies of states in the negative, neutral and positive charge state, respectively.

The energy of the four electrons of a neutral vacancy placed into the four $\mathrm{sp}^{3}$ hybrid orbitals is

$$
4 \bar{E}+6 \bar{J}-\frac{3 U}{2} .
$$

Estimates of the values of the parameters $[6,9,10]$ are reasonably consistent. All of the estimates of $\bar{E}$ derived from analytical integrations involving Slater or similar orbitals on the dangling bonds give values between -40 and $-30 \mathrm{eV} . \bar{J}$ ranges between 9.1 and $11 \mathrm{eV}$, with a value of $9.25 \mathrm{eV}$ favoured by Lannoo. The ratio $\Delta / U$, which determines the spacing of energies within each charge state, appears to have values near 1 in all of the 
calculations, but the values of $\Delta$ and $U$ separately vary more widely, between 2 and $11 \mathrm{eV}$ with values of $\Delta=4.3 \mathrm{eV}$ and $U=3.3 \mathrm{eV}$ being favoured by Lannoo.

Lowther [11] took an empirical approach similar to ours, but calculated the splitting parameter $(\Delta)$ separately for the neutral and negative vacancy. His values were, for $\mathrm{V}^{0}$, $\Delta=1.39 \mathrm{eV}$, and for $\mathrm{V}^{-}, \Delta=3.15 \mathrm{eV}$. The parameter that he calls $K$, which corresponds to Lannoo's $U / 4$ has the value $0.58 \mathrm{eV}$, and $\bar{J}$ he appears to take as zero. For these reasons, while his deductions for the neutral and negative vacancy are helpful, his model does not include any process which involves the positive or other charge states.

In the case of the charged vacancies, there is an additional component in the energy which arises because of the polarization of the lattice around the defect. All of the other energies and interactions involve the dangling-bond orbitals alone, but this polarization is of the surrounding lattice, not the dangling bonds. As such, it is quadratic in the charge in the simplest (linear response) models and therefore has the same value for negative and positive vacancies. Although we will treat this term as a purely empirical parameter, $e_{p o l}$, it is possible to estimate its value by considering the vacancy as a spherical cavity in a continuous dielectric medium, and calculating the polarization energy when this hole is charged. If the radius of this cavity is the nearest-neighbour distance in the diamond lattice, then

$$
e_{p o l}=3.8 \mathrm{eV} \text {. }
$$

This proves to be very close to the value that we obtain independently from our analysis of experimental data in section 4 .

\section{Experimental information}

The GR1 optical transition, at the neutral vacancy [1, 2, 12, 13], has a zero-phonon line at $1.673 \mathrm{eV}$, and is known to have a ${ }^{1} \mathrm{E}$ ground state and a ${ }^{1} \mathrm{~T}_{2}$ excited state. Although it has a large Jahn-Teller relaxation [3,7], one of the largest known, within this treatment the Jahn-Teller energy is smaller than the likely errors and will therefore not be taken into account. It is seen both in absorption and luminescence, but is not associated with any photoconductivity. There are higher-energy transitions, only seen in absorption [14], in the energy range 2.88-3.01 eV, which show hole photoconductivity [15] and which are the subject of a further paper [16].

The ND1 optical transition occurs at the negatively charged vacancy. Its ground state is ${ }^{4} \mathrm{~A}_{2}$ and its excited state is ${ }^{4} \mathrm{~T}_{1}$, with a zero-phonon line at $3.15 \mathrm{eV}$. It shows photoconductivity, assumed to be of electrons [17]. Its absorption intensity is $4.0 \pm 0.2$ times that of GR1 for the same concentration of defects [18].

The positive vacancy was identified in electron paramagnetic resonance (EPR)- the Baldwin defect [19], but Isoya et al [20] recently showed that the signal Baldwin identified in fact originates from the negative vacancy. Baldwin reported that light of $2.83 \mathrm{eV}$ ionized the neutral vacancies to produce this defect. He reported that the signal was stable for days in the absence of light at room temperature. However, Isoya himself has recently identified an EPR signal from a positive vacancy in type IIb, boron-doped diamond, and more detailed experiments are under way [21].

Electron, neutron or $\alpha$-particle damage produces vacancies in diamond, observed optically as GR1, ND1 or in EPR depending on the type of diamond that was irradiated. For example, in p-type diamond (type IIb, boron-doped samples) the GR1 centre is not seen for low radiation doses, but once a threshold dose corresponding to a vacancy concentration comparable with the boron concentration is exceeded [22], the GR1 centre appears. In 
diamonds which contain a high concentration of single substitutional nitrogen atoms (type $\mathrm{Ib}$ ), the ND1 centre is seen, although GR1 is often present at the same time, possibly due to inhomogeneity of the samples.

In diamond which contains appreciable concentrations of aggregated nitrogen (type Ia) both GR1 and ND1 are seen simultaneously, although the GR1 absorption is always stronger, and corresponds to a very much greater concentration of neutral than negative vacancies [18]. The A aggregate of nitrogen (a pair of substitutional atoms) is believed to have a donor level at about $4 \mathrm{eV}[23,24]$ or 'mid-gap' [25].

The purest type of diamond (type IIa), when irradiated, has negligible ND1 absorption, implying that its Fermi level is lower than in type Ia samples. Because pure diamond is a very good electrical insulator and the defect states tend to be deep and highly localized, it is possible that charged defects may be produced and survive for a long time before acquiring the equilibrium charge [26].

Most natural diamonds have rather inhomogeneous impurity concentrations. It is notable, though, that the GR1 band is seen in all but the most strongly doped samples, and $\mathrm{V}^{0}$ seems to be the most stable charge state except in these extremes of the IIb and Ib types.

The relevant difference in the diamond in all of these cases is just the Fermi energy $\left(E_{F}\right)$, which, located anywhere between the valence band edge $\left(E_{v}\right)$ and the conduction band edge $\left(E_{c}\right)$, stabilizes the different charge states of the vacancy. To summarize:

(i) in IIb diamond $\left(E_{F}=E_{v}+0.36 \mathrm{eV}\right), \mathrm{V}^{+}$is stable with respect to $\mathrm{V}^{0}$ or, in a convention used for semiconductors: $E(0 /+)>E_{v}+0.36 \mathrm{eV}$;

(ii) in Ia diamond $\left(E_{F}=E_{c}-4 \mathrm{eV}\right), \mathrm{V}^{0}$ is stable with respect to $\mathrm{V}^{+}$or $\mathrm{V}^{-}$, or $E(0 /+)$ $<E_{c}-4 \mathrm{eV}$ and $E(0 /-)>E_{c}-4 \mathrm{eV}$;

(iii) in IIa diamond $\left(E_{F}=E_{v}+2.75 \mathrm{eV}\right.$ ?), $\mathrm{V}^{0}$ is stable with respect to $\mathrm{V}^{+}$or $\mathrm{V}^{-}$or $E(0 /+)<E_{v}+2.75 \mathrm{eV}$ and $E(0 /-)>E_{v}+2.75 \mathrm{eV}$; and

(iv) in $\mathrm{Ib}$ diamond $\left(E_{F}=E_{c}-1.7 \mathrm{eV}\right), \mathrm{V}^{-}$is stable with respect to $\mathrm{V}^{0}$ or $E(0 /-)<E_{c}-1.7 \mathrm{eV}$.

We also interpret the presence of the GR1 in most irradiated diamond as implying that the vacancy is not a negative- $U$ centre, that is

$$
2 E^{0}<E^{+}+E^{-} \text {. }
$$

$\left(E^{+}, E^{0}, E^{-}\right.$are the energies of the ground state of the positively charged, neutral and negatively charged vacancy, respectively.) We have ignored lattice relaxation energies, since they are smaller than the uncertainties on the Fermi energies quoted here, and on all of the subsequent values quoted.

It is observed that the ${ }^{5} \mathrm{~A}_{2}$ state of the neutral vacancy, which can be seen in EPR work [27], has an anomalous temperature variation which could be due to transitions to another state which is close in energy to it (about $40 \mathrm{meV}$ ).

It is interesting to note that the irradiation of nitrogen-containing diamond (type I) leads to a larger concentration of vacancies near the nitrogen than distant from it, giving rise to a greater total concentration of vacancies in type I diamond than in purer samples (type IIa) [18]. Further, positron lifetime spectroscopy has shown that vacancy concentrations in boron-containing chemical-vapour-deposition diamond films are much smaller than in identical undoped films [28]. We suggest that this effect may be due to a charge-transfer process increasing the recombination of vacancies and interstitials during the growth process in p-type samples and inhibiting this effect where the Fermi level is higher, but this speculation is outside the scope of the present work. 


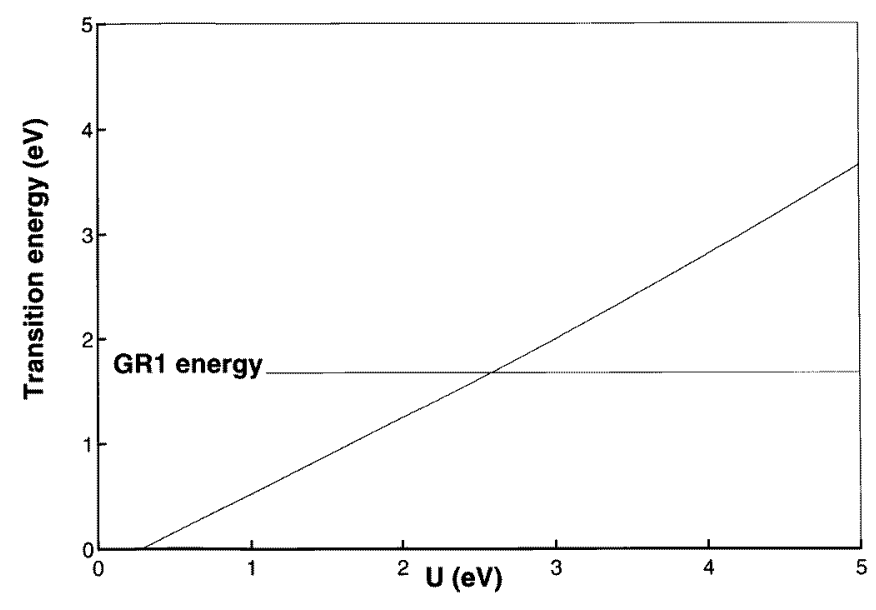

Figure 1. The energy of the ${ }^{1} \mathrm{E} \rightarrow{ }^{1} \mathrm{~T}_{2}$ transition (GR1) at the neutral vacancy as a function of the electron-electron energy parameter, $U$.

\section{Analysis of the energies}

If we look at the two transition energies in this model, in table 1, the ND1 transition is independent of $U$, and involves no CI, so we can use it to fix

$$
\Delta=3.15 \mathrm{eV} \text {. }
$$

Although the GR1 transition is independent of $\Delta$ to first order, CI mixes it in and figure 1 shows how the optical transition energy depends on $U$, and therefore

$$
U=2.6 \mathrm{eV} \text {. }
$$

With these values the energies of the states observed at the positive, neutral and negative vacancy can be calculated from the values in table 1 .

Taking the value of $\bar{J}$ derived by Lannoo of $9.25 \mathrm{eV}$, the ground-state energies of the three charge states become

$$
\begin{aligned}
& E^{+}\left({ }^{2} \mathrm{~T}_{2}\right)=3 \bar{E}-e_{p o l}+22.8 \mathrm{eV} \\
& E^{0}\left({ }^{1} \mathrm{E}\right)=4 \bar{E}+49.8 \mathrm{eV} \\
& E^{-}\left({ }^{4} \mathrm{~A}_{2}\right)=5 \bar{E}-e_{p o l}+86.2 \mathrm{eV}
\end{aligned}
$$

and therefore, using the conditions (i) to (iv),

$$
\begin{aligned}
& -32.7 \geqslant \bar{E}-e_{p o l}>-33.7 \mathrm{eV} \\
& -25.5>\bar{E}+e_{p o l}>-26.6 \mathrm{eV} .
\end{aligned}
$$

The negative- $U$ condition implies that

$$
0<e_{\text {pol }}<4.7 \mathrm{eV} \text {. }
$$

These expressions lead to limits which are considerably closer than the confidence that we have in the model or in the assumed parameters. We derive

$$
\begin{aligned}
& \bar{E}=-29.6 \pm 0.4 \mathrm{eV} \\
& e_{\text {pol }}=3.6 \pm 0.4 \mathrm{eV} .
\end{aligned}
$$


Note that the polarization energy derived here is very close to that predicted by equation (2.1). An alternative approach of using equation (2.1)'s value of the polarization energy $e_{p o l}$ to derive $\bar{E}$ and $\bar{J}$ gives values very close to those quoted-the model is acceptably self-consistent. The values derived are also quite close to Lannoo's.

Table 2. The energies of all of the electronic states of the positive, neutral and negative vacancies in diamond relative to the ground state of the neutral vacancy $\left({ }^{1} \mathrm{E}\right)$. Only the lowest-energy states of the doubly charged vacancy are shown.

\begin{tabular}{lll}
\hline Charge & State & Energies $(\mathrm{eV})$ \\
\hline $\mathrm{V}^{2+}$ & ${ }^{1} \mathrm{~A}_{1}$ & -0.6 \\
$\mathrm{~V}^{+}$ & ${ }^{2} \mathrm{~T}_{2}$ & $-1.0,3.3,6.5$ \\
$\mathrm{~V}^{+}$ & ${ }^{4} \mathrm{~T}_{1}$ & 1.2 \\
$\mathrm{~V}^{+}$ & ${ }^{2} \mathrm{E}$ & $1.8,6.6$ \\
$\mathrm{~V}^{+}$ & ${ }^{2} \mathrm{~T}_{1}$ & $2.6,5.5$ \\
$\mathrm{~V}^{+}$ & ${ }^{2} \mathrm{~A}_{1}$ & 3.8 \\
$\mathrm{~V}^{+}$ & ${ }^{4} \mathrm{~A}_{2}$ & 4.3 \\
$\mathrm{~V}^{0}$ & ${ }^{1} \mathrm{E}$ & $0.0,5.2,8.0$ \\
$\mathrm{~V}^{0}$ & ${ }^{3} \mathrm{~T}_{1}$ & $0.2,3.3,7.1$ \\
$\mathrm{~V}^{0}$ & ${ }^{1} \mathrm{~A}_{1}$ & $1.6,9.8$ \\
$\mathrm{~V}^{0}$ & ${ }^{1} \mathrm{~T}_{2}$ & $1.7,5.5,8.6$ \\
$\mathrm{~V}^{0}$ & ${ }^{5} \mathrm{~A}_{2}$ & 1.8 \\
$\mathrm{~V}^{0}$ & ${ }^{3} \mathrm{~A}_{2},{ }^{3} \mathrm{E},{ }^{3} \mathrm{~T}_{2},{ }^{1} \mathrm{~T}_{1}$ & 4.4 \\
$\mathrm{~V}^{-}$ & ${ }^{4} \mathrm{~A}_{2}$ & 3.2 \\
$\mathrm{~V}^{-}$ & ${ }^{2} \mathrm{~T}_{1}$ & $3.6,8.6$ \\
$\mathrm{~V}^{-}$ & ${ }^{2} \mathrm{E}$ & $4.7,7.7$ \\
$\mathrm{~V}^{-}$ & ${ }^{2} \mathrm{~T}_{2}$ & $4.9,8.3,11.2$ \\
$\mathrm{~V}^{-}$ & ${ }^{4} \mathrm{~T}_{1}$ & 6.4 \\
$\mathrm{~V}^{-}$ & ${ }^{2} \mathrm{~A}_{1}$ & 9.0 \\
$\mathrm{~V}^{2-}$ & ${ }^{1} \mathrm{~T}_{2}$ & 8.8 \\
\hline
\end{tabular}

Since the energy zero in this treatment is not very significant, we have quoted all of the rest of the vacancy energies relative to the energy of the ground state of the neutral vacancy $\left(E^{0}\left({ }^{1} \mathrm{E}\right)=-68.3 \mathrm{eV}\right)$.

Table 2 gives the energies of all of the configurations of the vacancy relative to this. Figure 2 shows the energies of the states with respect to the Fermi energy - that is, the energy of the vacancy in that state with an electron or hole at the Fermi energy to balance the charge.

\section{Discussion and implications}

\subsection{Stability of states}

Within this model, the stable charge state of the vacancy is positive $\left({ }^{2} T_{2}\right)$ if the Fermi energy is below about $1.0 \mathrm{eV}$ above the valence band, neutral $\left({ }^{1} \mathrm{E}\right)$ if $1.0<E_{F}<3.2 \mathrm{eV}$ and negative $\left({ }^{4} \mathrm{~A}_{2}\right)$ otherwise. Or, in other terms, $E(0 /+)=E_{v}+1 \mathrm{eV}$, and $E(0 /-)=E_{v}+3.2$ $\mathrm{eV}$. The doubly charged positive and the doubly charged negative vacancies have rather higher energies even when the polarization term, $-4 e_{p o l}$ (which is quadratic in the charge), is included. When the Fermi level is at the valence or conduction band edge, respectively, the doubly positive and negative charged states are 0.4 and $0.1 \mathrm{eV}$ higher than the singly charged states, respectively. Equivalently, $E(+/++)=E_{v}-0.4 \mathrm{eV}$ and $(E-/--)=E_{c}+0.1 \mathrm{eV}$. Although the stabilities of $\mathrm{V}^{-}, \mathrm{V}^{0}$ and $\mathrm{V}^{+}$are very similar in this model to the calculations 


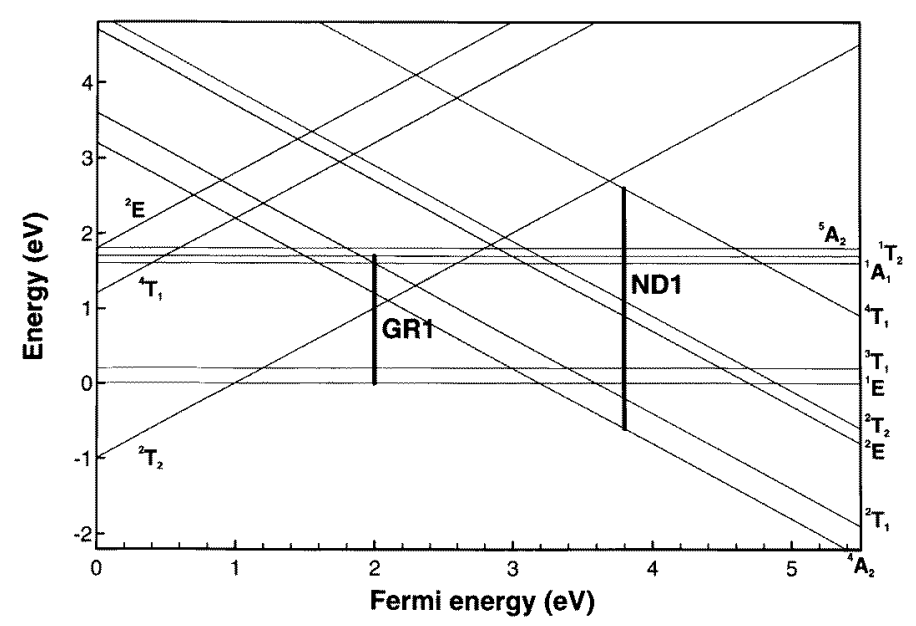

Figure 2. Energies of the lower states of the vacancy plotted against the position of the Fermi energy in the $5.5 \mathrm{eV}$ bandgap of diamond: $\mathrm{V}^{+}+e_{F}, \mathrm{~V}^{0}$ and $\mathrm{V}^{-}-e_{F}$, against $e_{F}$. Therefore, the $\mathrm{V}^{+}$states have positive gradients and the $\mathrm{V}^{-}$states have negative gradients.

of Bernholc et al [8], they predicted that $\mathrm{V}^{2+}$ and $\mathrm{V}^{2-}$ would be stable when the Fermi level was close to the valence and conduction band edge, respectively.

Both of the negative- $U$ processes

$$
\begin{aligned}
& 2 \mathrm{~V}^{-} \rightarrow \mathrm{V}^{2-}+\mathrm{V}^{0} \\
& 2 \mathrm{~V}^{+} \rightarrow \mathrm{V}^{2+}+\mathrm{V}^{0}
\end{aligned}
$$

are energetically unfavourable by 2.4 and $1.2 \mathrm{eV}$ respectively. The $\mathrm{V}^{2-}$ and $\mathrm{V}^{2+}$ charge states are unlikely to be observed.

It is observed that in type IaA diamond, GR1 and ND1 bands coexist, and after annealing at $600-800{ }^{\circ} \mathrm{C}$ these come into equilibrium corresponding to an intensity ratio of about 10:1 [18]. Assuming a Boltzmann distribution, there will be an energy difference between $\mathrm{V}^{0}+e_{F}$ and $\mathrm{V}^{-}$of about about $0.2 \mathrm{eV}$. If, as was discussed earlier, the donor level is 'mid-gap', figure 2 shows that our model will give approximately this energy difference.

Other states of the vacancy have been discussed or observed, as described below.

(i) All of the theoretical models of the vacancy, from that of Coulson and Kearsley onwards $[6,9]$, predicted that the ${ }^{3} \mathrm{~T}_{1}$ state had a low energy, but it has never been observed. Mainwood et al [29], in the course of investigating a distorted vacancy-like defect, gave limits on the energy of ${ }^{3} \mathrm{~T}_{1}$ as between 0.04 and at $0.2 \mathrm{eV}$ above the ${ }^{1} \mathrm{E}$ ground state. Our model positions ${ }^{3} \mathrm{~T}_{1}$ at $0.2 \mathrm{eV}$ above ${ }^{1} \mathrm{E}$, although the errors on the position of both of these states are of the same magnitude.

(ii) The ${ }^{5} \mathrm{~A}_{2}$ state of the neutral vacancy is produced by ionization of one of the $\mathrm{V}^{-}$ states, by UV light [27]. The situation is complicated with some rather strange temperature effects, but it appears that the ${ }^{5} \mathrm{~A}_{2}$ level is populated via the excited state of ND1 $\left({ }^{4} \mathrm{~T}_{1}\right)$, in that UV light of about the ND1 energy is required and GR1 absorption is enhanced at the same time:

$$
E^{-}\left({ }^{4} \mathrm{~A}_{2}\right)+h v \rightarrow E^{-}\left({ }^{4} \mathrm{~T}_{1}\right) \rightarrow E^{0}\left({ }^{5} \mathrm{~A}_{2}\right)+e_{F} .
$$

This requires that the electron is transferred to a defect (in these samples, one of the high concentration of nitrogen aggregates) with an energy level less than $4.6 \mathrm{eV}$ above the valence 
band edge. We predict from this model that it may be impossible to populate $\left({ }^{5} \mathrm{~A}_{2}\right)$ in very pure type IIa samples where the vacancies are likely to be much more remote from the nitrogen defects.

The ${ }^{5} \mathrm{~A}_{2}$ state is observed to have a temperature dependence consistent with decay to another state about $0.04 \mathrm{eV}$ higher in energy than it [27]. In this model, the ${ }^{1} \mathrm{~T}_{2}$ is $0.1 \mathrm{eV}$ below ${ }^{5} \mathrm{~A}_{2}$, well within the expected errors. However, the transition from ${ }^{5} \mathrm{~A}_{2}$ to ${ }^{1} \mathrm{~T}_{2}$ is forbidden (because they are different spin states), even when the small spin-orbit coupling of the states is included. The same is true of the ${ }^{1} \mathrm{~A}_{1}$ state $0.2 \mathrm{eV}$ below ${ }^{5} \mathrm{~A}_{2}$, and the other states into which it could decay $\left(\mathrm{a}^{3} \mathrm{~T}_{1}\right.$ state) are about $1.5 \mathrm{eV}$ below it or even further away. We have no explanation for the state influencing the decay, unless it is associated with another defect or impurity.

(iii) There is no ND1 luminescence, which implies that the excited state $\left({ }^{4} \mathrm{~T}_{1}\right)$ is unstable with respect to one of the $\mathrm{V}^{0}$ states. A Born-Haber cycle:

$$
E^{-}\left({ }^{4} \mathrm{~T}_{1}\right) \rightarrow E^{0}\left({ }^{3} \mathrm{~T}_{1}\right)+\mathrm{e}_{c}
$$

shows that this transition would take place releasing $0.7 \mathrm{eV}$, and an electron into the conduction band $\left(\mathrm{e}_{c}\right)$.

\subsection{Photoconductivity}

There is no photoconductivity associated with GR1, so the ${ }^{1} \mathrm{~T}_{2}$ state must be stable against losing an electron to the conduction band, and against capturing an electron from the valence band to produce $\mathrm{a} \mathrm{V}^{+}$and $\mathrm{V}^{-}$state, respectively. In this model, these processes are both endothermic.

On the other hand, the higher-energy transitions at the neutral vacancy, GR2-8, whose absorption lines are at $2.88-3.01 \mathrm{eV}$, are associated with hole photoconductivity. Although there is still some confusion over the GR2-8 excited state (but see [16]), the energies here show that it is quite possible for a photoconductive process to take place, though not one which involves a $\mathrm{V}^{-}$final state.

ND1 is also photoconductive [17], and in this model the excited state $\left({ }^{4} \mathrm{~T}_{1}\right)$ would lose an electron to the conduction band:

$$
E^{-}\left({ }^{4} \mathrm{~T}_{1}\right) \rightarrow E^{0}\left({ }^{3} \mathrm{~T}_{1}\right)+\mathrm{e}_{c}
$$

as well as populating the $\mathrm{V}^{0}\left({ }^{5} \mathrm{~A}_{2}\right)$ state. Other final states of $\mathrm{V}^{0}$ would involve a spinforbidden transition, and therefore would be much less likely. This is consistent with the observations of Dyer and Du Preez [30], who found that intense illumination in the ND1 band reduced its intensity while enhancing the GR1 absorption, and heating in the dark reversed these changes, as equilibrium between charge states was restored. The ND1 transition has a sharp zero-phonon line, which implies that neither the initial nor the final states are in a band. This can be seen to be easily possible from figure 2 , although we cannot determine precisely where the band edges occur. This observation is fully consistent with the observed photoconductivity.

The ${ }^{5} \mathrm{~A}_{2}$ state of the neutral vacancy is not photoconductive even when illuminated by $3.4 \mathrm{eV}$ photons [27]. In this model its energy is very close to the $\mathrm{V}^{0}\left({ }^{1} \mathrm{~T}_{2}\right)$ state, so by the same arguments, no photoconductivity would be expected from this state. However, when it is illuminated by $3.4 \mathrm{eV}$ light, one would expect that ionization of some kind would be possible, producing either holes in the valence band $\left(\mathrm{h}_{v}\right)$ or electrons in the conduction band. There could be two processes, as described below. 
(a) Releasing holes into the valence band:

$$
E^{0}\left({ }^{5} \mathrm{~A}_{2}\right)+h v \rightarrow E^{-}+\mathrm{h}_{v} .
$$

Even the lowest-energy state of the negative vacancy has too high an energy for this process to take place.

(b) Releasing electrons into the conduction band:

$$
E^{0}\left({ }^{5} \mathrm{~A}_{2}\right)+h v \rightarrow E^{+}+\mathrm{e}_{c} .
$$

For this transition to be allowed by spin, the final state would have to be $\mathrm{V}^{+}\left({ }^{4} \mathrm{~T}_{1}\right)$, whose energy of $2.4 \mathrm{eV}$ would also be too high for the process to occur, although some photoionization may come from the spin-forbidden transition into the $\mathrm{V}^{+}$ground state.

Photoionization of the neutral vacancy involves a balance between the two spin-allowed processes, with non-radiative decay into the normal ground states:

$$
E^{0}\left({ }^{1} \mathrm{E}\right)+h v \rightarrow E^{-}\left({ }^{2} \mathrm{~T}_{1}\right)+\mathrm{h}_{F}
$$

and

$$
E^{-}\left({ }^{4} \mathrm{~A}_{2}\right)+h v \rightarrow E^{0}\left({ }^{3} \mathrm{~T}_{1}\right)+\mathrm{e}_{F}
$$

with the hole $\left(\mathrm{h}_{F}\right)$ and electron at the Fermi level. Using the energies of table 2, the Fermi level would be above the conduction band for the first process to be more favourable than the second. We therefore expect that it would be possible to photoionize $\mathrm{V}^{-}$to $\mathrm{V}^{0}$ but not the reverse, just as Dyer and Du Preez observed [30]. The former of these processes is the one Baldwin observed [19, 20], using $2.8 \mathrm{eV}$ excitation.

\subsection{Optical transitions at the vacancy}

Because we now have not only the energies but also approximate many-electron wavefunctions for the vacancy states, we can calculate the transition probabilities for the optical transitions observed. The ND1 intensity, I(ND1), is proportional to the matrix element $\left\langle{ }^{4} \mathrm{~A}_{2}|r|^{4} \mathrm{~T}_{1}\right\rangle$, squared, where $r=(x, y, z)$ is the dipole operator. If the $\mathrm{sp}^{3}$ hybrids which make up the dangling bonds are expressed as a linear combination of atomic orbitals (LCAO) on the four atoms surrounding the vacancy [6] and the overlap between different atoms is neglected, then the matrix elements of the dipole operator $\langle\mathrm{a}|r| \mathrm{a}\rangle=0$, and $\langle\mathrm{t}|r| \mathrm{t}\rangle=0$, whichever of the three t components $(x, y$, or $z)$ are chosen. So,

$$
I(\mathrm{ND} 1) \propto\left|\left\langle{ }^{4} \mathrm{~A}_{2}|r|^{4} \mathrm{~T}_{1}\right\rangle\right|^{2}=|\langle\mathrm{a} \overline{\mathrm{a}} x y z|r| \mathrm{a} x \bar{x} y z\rangle|^{2}=|\langle\mathrm{a}|r| x\rangle|^{2} .
$$

The GR1 transition is not so simple, since if we look only at the lowest ${ }^{1} \mathrm{E}$ and ${ }^{1} \mathrm{~T}_{2}$ states, the matrix elements are all of the type $\langle a \bar{a} x \bar{x}|r| \mathrm{a} \bar{x} x \bar{y}\rangle$ which are in effect two-electron transitions, and therefore zero for an electric dipole transition. However, both ground and excited states involve configuration interaction:

$$
\begin{gathered}
\left|{ }^{1} \mathrm{E}\right\rangle=\frac{\alpha}{\sqrt{6}}(2|\mathrm{a} \overline{\mathrm{a}} x \bar{x}\rangle-|\mathrm{a} \overline{\mathrm{a}} y \bar{y}\rangle-|\mathrm{a} \overline{\mathrm{a}} z \bar{z}\rangle)+\frac{\beta}{2}(|\mathrm{a} \overline{x y} z\rangle+|\overline{\mathrm{a}} x y \bar{z}\rangle-|\mathrm{a} \bar{x} y \bar{z}\rangle-|\overline{\mathrm{a}} x \bar{y} z\rangle) \\
+\frac{\gamma}{\sqrt{6}}(2|y \bar{y} z \bar{z}\rangle-|x \bar{x} z \bar{z}\rangle-|x \bar{x} y \bar{y}\rangle)
\end{gathered}
$$

with the constants $\alpha, \beta$ and $\gamma$ determined by Lannoo's configuration interaction matrix. An expression for $\left|{ }^{1} \mathrm{~T}_{2}\right\rangle$ can be derived in a similar way, also subject to CI. The intensity of the transition is proportional to the square of the matrix element, and is a clear measure of the strength of the configuration interaction in these states:

$$
I(\mathrm{GR} 1) \propto\left|\left\langle{ }^{1} \mathrm{E}|r|{ }^{1} \mathrm{~T}_{2}\right\rangle\right|^{2}=0.115|\langle\mathrm{a}|r| x\rangle|^{2} .
$$


Hence the intensity in the ND1 would be expected to be a factor of 8.7 greater than that of the GR1 for the same number of defects. This can be compared with the experimental ratio of $4.0 \pm 0.2$ [18]. Considering the drastic approximations used (see, e.g., reference [31]), and the fact that it is far easier to get energies right than wavefunctions, the agreement is reasonable.

At the neutral vacancy, apart from the GR1 and GR2-8 transitions, it may be possible to see absorption at the ${ }^{1} \mathrm{E}$ to ${ }^{1} \mathrm{~T}_{1}$ transition at around $4.4 \mathrm{eV}$ in type IIa diamonds. There are several possible absorption or cathodoluminescence lines listed [24, 32], but there are insufficient data available to make a positive identification.

This model, like many more before it, predicts the ${ }^{3} \mathrm{~T}_{1}$ state close above the ground state of the neutral vacancy. It should be possible to populate it, either by photoionization of $\mathrm{V}^{-}$or thermally, and to detect its spin resonance.

The positive vacancy, with a ${ }^{2} \mathrm{~T}_{2}$ ground state, will have a Jahn-Teller relaxation, which this model makes no attempt to include in its energy. The position of the valence band edge relative to these many-electron states cannot be determined with any accuracy. If the $\mathrm{V}^{+}$ ground state were within the valence band, neither EPR nor sharp optical signals would be seen. Isoya's recent observation [21] of a positive-vacancy EPR signal would suggest that in his sample, at least, the ground state is in the energy gap. We would expect, in this case, that it might be possible to see the three optical transitions: ${ }^{2} \mathrm{~T}_{2}$ to ${ }^{2} \mathrm{E}$, at about $2.8 \mathrm{eV}$, ${ }^{2} \mathrm{~T}_{2}$ to ${ }^{2} \mathrm{~T}_{1}$, at about $3.6 \mathrm{eV}$, and ${ }^{2} \mathrm{~T}_{2}$ to ${ }^{2} \mathrm{~A}_{1}$, at about $4.8 \mathrm{eV}$. Other transitions would be forbidden by spin, by symmetry, or would be within the bands.

\subsection{Charge states of other defects}

It was assumed in the original expressions of the stability of defects that

$$
\mathrm{V}^{0}+\mathrm{N}^{0} \rightarrow \mathrm{V}^{-}+\mathrm{N}^{+}
$$

and

$$
\mathrm{V}^{0}+\mathrm{B}^{0} \rightarrow \mathrm{V}^{+}+\mathrm{B}^{-}
$$

are both exothermic, and with the ionization energies of nitrogen and boron known, these processes release 0.6 and $1.4 \mathrm{eV}$ respectively. The latter process is the one observed when irradiated p-type diamond fails to show a GR1 absorption until the irradiation is high enough for all of the acceptors to have been compensated [22]. Other impurities with donor or acceptor energies less than 2.3 or $1 \mathrm{eV}$, respectively, may show similar charge-transfer processes. These have not been observed, although a few defects are known in two charge states: $\mathrm{H} 3$ and $\mathrm{H} 2$ [33], the nitrogen interstitial [34], and the nitrogen-vacancy complex [35], for example.

\subsection{Formation energy for the vacancy}

A very simple calculation for the formation energy of the vacancy [36] $\left(E_{v a c}\right)$ compares the energy of the four dangling bonds in the defect with the energy reclaimed when the displaced atom reaches the surface, reforming two bonds. Taking the surface bond energy as the energy of carbon bonds broken when the surface graphitizes [37] (350 $\pm 50 \mathrm{~kJ} \mathrm{~mol}^{-1}$ per bond),

$$
E_{v a c}=8.5 \pm 1.0 \mathrm{eV} \text {. }
$$

This is similar to other recently obtained values, for example Bernholc's value of $7.2 \mathrm{eV}$, plus $0.25 \mathrm{eV}$ Jahn-Teller energy [8]. 


\section{Conclusions}

Over the years, a wealth of data have been generated for the vacancy in diamond. Yet some basic questions remain unanswered, like that of the 'position in the gap' (actually the relative energies of the different charge states). We have used a simple model of the many-electron interactions at the vacancy in diamond to construct the energies of all of the neutral, negative and positively charged vacancy states. This gives a framework within which to analyse the relative stability of these charge states. This proves successful: the photoconductivity and photoionization of these states, or lack of it, then agree with almost all of the experimental data now available.

We are still unable to account for the temperature dependence of the ${ }^{5} \mathrm{~A}_{2}$ state of the neutral vacancy. The higher-energy optical transitions at the neutral vacancy, GR2-8, will be discussed in a further paper [16].

\section{Acknowledgments}

We would like to thank Mark Newton, Gordon Davies and Michel Lannoo for helpful discussions and comments.

\section{References}

[1] Davies G (ed) 1994 Properties of Diamond, a Data Review (Stevenage: IEE)

[2] Davies G 1981 Rep. Prog. Phys. 44787

[3] Stoneham A M 1977 Solid State Commun. 21339

[4] Newton M E 1994 Properties of Diamond, a Data Review ed G Davies (Stevenage: IEE) p 153 Newton M E 1994 Properties of Diamond, a Data Review ed G Davies (Stevenage: IEE) p 166

[5] See, for example, Breuer S J and Briddon P R 1995 Phys. Rev. B 516984

[6] Coulson C A and Kearsley M J 1957 Proc. R. Soc. A 241433

[7] Lannoo M and Stoneham A M 1968 J. Phys. Chem. Solids 291987

[8] Bernholc J, Antonelli A, Del Sole T M, Bar-Yam Y and Pantelides S T 1988 Phys. Rev. Lett. 612689

[9] Lannoo M and Bourgoin J 1981 Point Defects in Semiconductors, I: Theoretical Aspects (Berlin: Springer) pp 141-54

[10] Coulson C A and Larkins F P 1971 J. Phys. Chem. Solids 322245

[11] Lowther J E 1993 Phys. Rev. 4811592

[12] Davies G and Penchina C 1974 Proc. R. Soc. A 338359

[13] Walker J 1979 Rep. Prog. Phys. 421605

[14] Davies G 1977 Nature 269498

[15] Farrer R G and Vermeulen L A 1972 J. Phys. C: Solid State Phys. 52762

[16] Stoneham A M and Mainwood A 1997 Shallow Levels in Semiconductors ed C A J Ammerlaan and B Pajot (Singapore: World Scientific) p 165

[17] Vermeulen L A, Clark C D and Walker J 1975 Lattice Defects in Semiconductors 1974 (Inst. Phys. Conf. Ser. 23) ed F A Huntley (Bristol: Institute of Physics Publishing) p 274

Walker J, Vermeulen L A and Clark C D 1974 Proc. R. Soc. A 341253

[18] Davies G, Lawson S C, Collins A T, Mainwood A and Sharp S J 1992 Phys. Rev. B 4613157

[19] Baldwin J A 1963 Phys. Rev. Lett. 10220

Loubser J H N and van Wyk J A 1978 Rep. Prog. Phys. 411201

[20] Isoya J, Kanda H, Uchida Y, Lawson S C, Yamasaki S, Itoh H and Morita Y 1992 Phys. Rev. B 451436

[21] Newton M E 1996 private communication

[22] Collins A T 1977 Radiation Effects in Semiconductors 1976 (Inst. Phys. Conf. Ser. 31) ed N B Urli and J W Corbett (Bristol: Institute of Physics Publishing) p 346

Dyer H B and Fernando P 1966 Br. J. Appl. Phys. 17419

[23] Denham P, Lightowlers E C and Dean P J 1967 Phys. Rev. 161762

[24] Davies G 1976 Chem. Phys. Carbon 131 
[25] Briddon P R, Heggie M I and Jones R 1992 Mater. Sci. Forum 83-87 457

[26] Henderson B 1991 private communication

[27] van Wyk J A, Tucker O D, Newton M E, Baker J M, Woods G S and Spear P 1995 Phys. Rev. B 5212657

[28] Dannefaer S, Zhu W, Bretagnon T and Kerr D 1996 Phys. Rev. B 531979

[29] Mainwood A, Lowther J E and van Wyk J A 1993 J. Phys.: Condens. Matter 57929

[30] Dyer H B and Du Preez L 1965 J. Chem. Phys. 421898

[31] Stoneham A M 1966 Proc. Phys. Soc. 88135

[32] Clark C D, Collins A T and Woods G S 1992 Properties of Natural and Synthetic Diamond ed J E Field (London: Academic) p 35

[33] Lawson S C, Davies G, Collins A T and Mainwood A 1992 J. Phys.: Condens. Matter 43439

[34] Kiflawi I, Mainwood A, Kanda H and Fisher D 1996 Phys. Rev. B 5416719

[35] Loubser J H N and van Wyk J A 1977 Diamond Research vol 77 (London: Industrial Diamond Information Bureau) p 11

[36] Stoneham A M 1992 Properties of Natural and Synthetic Diamond ed J E Field (London: Academic) p 3

[37] Evans S 1994 Properties of Diamond, a Data Review ed G Davies (Stevenage: IEE) p 68 